\title{
Benralizumab does not impair antibody response to seasonal influenza vaccination in adolescent and young adult patients with moderate to severe asthma: results from the Phase IIIb ALIZE trial
}

This article was published in the following Dove Press journal: Journal of Asthma and Allergy

\author{
Pamela L Zeitlin' \\ Mila Leong ${ }^{2}$ \\ Jeremy Cole ${ }^{3}$ \\ Raburn M Mallory ${ }^{4}$ \\ Vivian $\mathrm{H}$ Shih $^{5}$ \\ Richard F Olsson ${ }^{6}$ \\ Mitchell Goldman ${ }^{5}$
}

On behalf of the ALIZE study investigators

'Department of Pediatrics, National Jewish Health, Denver, CO, USA; ${ }^{2}$ Pediatric Pulmonary and Asthma Associates, Northfield, NJ, USA; ${ }^{3} \mathrm{OK}$ Clinical Research, LLC, Edmond, OK, USA; ${ }^{4}$ Medlmmune LLC, Gaithersburg, MD, USA; ${ }^{5}$ AstraZeneca, Gaithersburg, MD, USA; ${ }^{A}$ AstraZeneca, Gothenburg, Sweden
Correspondence: Pamela $L$ Zeitlin Department of Pediatrics, National Jewish Health, 1400 Jackson Street, Denver, CO 80206 , USA

Tel +l 8772255654

Email pzeitli@jhmi.edu
Background and objectives: Benralizumab is a humanized, afucosylated monoclonal antibody against the IL-5R $\alpha$. Initial monthly followed by every-other-month injections result in rapid and nearly complete eosinophil depletion. We evaluated whether three doses of benralizumab modifies antibody response to seasonal influenza vaccination for adolescent/young adult patients with moderate to severe asthma.

Methods: ALIZE (NCT02814643) was a Phase IIIb randomized controlled trial of patients aged 12-21 years receiving medium- to high-dosage inhaled corticosteroids/long-acting $\beta_{2}$-agonists. Patients received benralizumab $30 \mathrm{mg}$ or placebo at Weeks 0 , 4, and 8 , plus tetravalent influenza vaccination at Week 8 . At Week 12, strain-specific antibody responses following vaccination were assessed for four influenza antigens by hemagglutination inhibition (HAI) and microneutralization $(\mathrm{MN})$ assays.

Results: A total of 103 patients were randomized and received benralizumab $(n=51)$ or placebo $(\mathrm{n}=52)$. There were no consistent differences in HAI or MN antibody responses at Week 12 between patients receiving benralizumab or placebo. HAI geometric mean fold rises (GMFRs) for all influenza strains tested were 3.3-4.2 for benralizumab vs 3.4-3.9 for placebo; MN GMFRs were 2.8-5.1 for benralizumab vs 3.2-4.4 for placebo. A $\geq 4$-fold rise in HAI from Weeks 8 to 12 occurred in $44.0 \%-56.0 \%$ and $30.6 \%-49.0 \%$ of patients receiving benralizumab and placebo, respectively. At Week $12,78.0 \%-100 \%$ vs $79.6 \%-100 \%$ of patients receiving benralizumab and placebo, respectively, achieved $\mathrm{a} \geq 40 \mathrm{HAI}$ antibody titer. There were no significant safety findings. Conclusion: Benralizumab did not impair the antibody response to seasonal virus vaccination in adolescents and young adult patients with moderate to severe asthma.

Keywords: benralizumab, interleukin-5, interleukin-5 receptor, influenza vaccination, eosinophils, antibody response, asthma

\section{Plain language summary}

Eosinophils are white blood cells that play an important role in the body's immune system. Patients with asthma often have an increased eosinophil count in their blood and airways, contributing to inflammation and other asthma symptoms. Benralizumab is approved for patients with uncontrolled asthma despite moderate- to high-dosage asthma treatments, and who also have increased eosinophil counts. Because eosinophils may potentially also play a role in the immune system's response to vaccination, we tested whether benralizumab modifies the response to the flu (influenza) vaccine. Vaccines work by making the body produce antibodies. We assessed the immune response by measuring the blood concentration of antibodies specific to the flu vaccine. 
The ALIZE study was designed to measure this effect during the 2016-2017 flu season in patients aged 12-21 years with moderate to severe asthma. We found that the responses to the flu vaccine were equivalent between patients who received benralizumab and those who received a control injection with no therapeutic action. Therefore, treatment with benralizumab does not affect the immune system's response to flu vaccination.

\section{Introduction}

Benralizumab (Fasenra ${ }^{\mathrm{TM}}$ ) is a humanized, IL-5R $\alpha$-directed monoclonal antibody that induces rapid and nearly complete eosinophil depletion. ${ }^{1}$ Eosinophils are the dominating effector cells associated with asthma; therefore, inhibition of eosinophilic inflammation or eosinophilia is expected to decrease airway injury, mucus hypersecretion, and bronchial hyper-responsiveness. ${ }^{1,2}$ In clinical studies, benralizumab treatment resulted in nearly complete depletion of blood and airway eosinophils for patients with mild to severe asthma. ${ }^{3,4}$ In the SIROCCO (NCT01928771) and CALIMA (NCT01914757) Phase III clinical trials, treatment with benralizumab significantly reduced exacerbation frequency and improved lung function and disease control compared with placebo for patients aged 12-75 years with severe, uncontrolled eosinophilic asthma receiving high-dosage inhaled corticosteroids (ICS)/long-acting $\beta_{2}$-agonists (LABA), ${ }^{5,6}$

In the USA, benralizumab is indicated for add-on maintenance treatment of patients with severe asthma aged 12 years and older, and with an eosinophilic phenotype. ${ }^{7}$ In Europe, benralizumab is indicated for add-on maintenance treatment of adult patients with severe eosinophilic asthma that is inadequately controlled despite treatment with highdosage ICS/LABA. ${ }^{8}$ In Japan, benralizumab is indicated for add-on treatment of patients with bronchial asthma who continue to experience asthma exacerbations despite treatment with high-dosage ICS and other asthma controllers. ${ }^{9}$

Influenza has been linked with the development of asthma and acute exacerbations for both adults and children with asthma. ${ }^{10}$ Asthma-associated chronic airway inflammation and type two immune response contribute to reduced respiratory tract antiviral immunity for patients with asthma. ${ }^{11}$ Consequently, patients with asthma are also at increased risk of developing influenza-related complications, compared with the general population. ${ }^{11}$ Although the available evidence is mostly from observational studies, influenza vaccination has been associated with a reduced rate of asthma exacerbations, influenza complications, and health care resource utilization. ${ }^{11}$ Because benralizumab is an immunomodulator, it is important to determine whether it influences immune system functioning, including response to influenza vaccination in this at-risk population.

Benralizumab is an afucosylated, immunoglobulin $\mathrm{G} 1_{\mathrm{K}}$ monoclonal antibody that binds specifically to the human IL-5R $\alpha,{ }^{1}$ which is expressed almost exclusively on the surface of eosinophils and basophils. ${ }^{12}$ IL-5R $\alpha$ solely binds IL-5, a key cytokine involved in regulating the differentiation, proliferation, and activation of eosinophils. The afucosylation of benralizumab increases its ability to bind to human Fc $\gamma$ RIIIa, the main $\mathrm{Fc}$ receptors expressed on natural killer cells, macrophages, and neutrophils. Benralizumab activates these effector cells upon binding to IL-5R $\alpha$ expressed by eosinophils, leading to their rapid apoptosis via antibody-dependent cell-mediated cytotoxicity. ${ }^{1}$ Eosinophils are effector cells with additional important roles in the regulation of local immunity and tissue remodeling and repair. ${ }^{13}$ They also may have a role in the immune response associated with viral vaccination and infection, either by playing a fundamental role in the innate host viral response or as a component of the acquired immune response..$^{13}$ Therefore, it is important to determine whether the reduction in eosinophils resulting from benralizumab treatment influences the functioning of the immune system following vaccination.

The aim of this Phase IIIb trial (ALIZE; NCT02814643) was to evaluate the efficacy, safety, pharmacokinetics (PK), and immunogenicity of a fixed dose of benralizumab on the humoral immune response following seasonal influenza virus vaccination for adolescent/young adult patients with moderate to severe asthma and an eosinophilic phenotype.

\section{Ethics approval and informed consent}

An Independent Ethics Committee or Institutional Review Board, as applicable, approved the final clinical study protocol for each study center. The study was performed in accordance with the Declaration of Helsinki and the International Council for Harmonisation/Good Clinical Practice guidelines, applicable regulatory requirements, and the AstraZeneca policy on bioethics. All patients, or parents/ legal guardians for those aged $<18$ years, provided written informed consent to participate in the study.

\section{Methods \\ Study design}

ALIZE (NCT02814643) was a Phase IIIb randomized, double-blind, parallel-group, placebo-controlled trial of patients aged 12-21 years receiving medium- to high-dosage 
ICS/LABA (Figure 1). The study was conducted at 30 study sites in the USA. The final clinical study protocol for each study center was approved by a central Institutional Review Board (Schulman Associates, Institutional Review Board, Inc., 4445 Lake Forest Drive, Cincinnati, OH, USA).

At Visit 1, patients were assessed for confirmation of physician-diagnosed asthma and degree of disease severity based on background medication use. For inclusion in the study, patients were required to have a documented history of asthma and current, regular use of ICS prior to enrollment. At Visit 2, patients were evaluated to determine whether they fulfilled the following inclusion criteria: current treatment with ICS and LABA (ICS dosage $\geq 500 \mu \mathrm{g}$ /day fluticasone propionate dry powder formulation or equivalent/day), morning prebronchodilator forced expiratory volume in 1 second $\left(\mathrm{FEV}_{1}\right)$ of $>50 \%$ predicted, weight $\geq 40 \mathrm{~kg}$, and uncontrolled asthma. Uncontrolled asthma was defined as one or more of the following: Asthma Control Questionnaire 6 [ACQ-6] score $\geq 1.5$, peak expiratory flow of $60 \%-80 \%$ predicted, $\geq 1$ exacerbation requiring oral corticosteroids in the previous year, and/or persistent symptoms in the previous 2-4 weeks. Exclusion criteria included the following: clinically important pulmonary disease other than asthma, major comorbidity, history of Guillain-Barré syndrome, smoking, pregnancy, known immunodeficiency disorder or history of cancer, allergy to eggs, use of immunosuppressive medication, previous treatment with benralizumab, previous treatment with other biologic (within 4 months or five drug half-lives, whichever was longer), treatment with nonbiological investigational drug (within 30 days or five drug half-lives, whichever was longer), receipt of live attenuated vaccine within 30 days of randomization, receipt of an influenza vaccine within 90 days of randomization, and asthma exacerbation or other illness at randomization.

Adolescents (aged 12-<18 years) and young adults (aged 18-21 years) were randomized 1:1 to receive benralizumab $30 \mathrm{mg}$ by subcutaneous injection or placebo at Weeks 0,4 , and 8 (as per the indicated dosage of $30 \mathrm{mg}$ every 4 weeks for the first three doses, then every 8 weeks). ${ }^{7,8}$ Patients received one dose of quadrivalent influenza vaccine (FluLaval ${ }^{\circledR}$ Quadrivalent; GlaxoSmithKline plc, Brentford, UK) intramuscularly at Week 8 , when benralizumab concentration would have reached steady state. ${ }^{7,8}$ This vaccine was formulated to contain $15 \mu \mathrm{g}$ of hemagglutinin (HA) for each of the following viruses: A/California/7/2009 (H1N1); A/Hong Kong/4801/2014 (H3N2); B/Phuket/3073/2013 (Yamagata lineage); and B/Brisbane/60/2008 (Victoria lineage). Blood samples for evaluation of antibody response were drawn at Week 8 (prior to vaccine administration), to determine baseline antibody concentrations, and Week 12 (study end), to determine the antibody response to influenza virus. All patients who completed the double-blind, randomized treatment period were followed for an additional 8 weeks until a follow-up visit at Week 20. Blood samples for eosinophil counts, PK, and safety analyses were also drawn at Week 0 and Week 20.

\section{Outcomes}

The primary outcome, effect of benralizumab on the humoral immune response following seasonal influenza
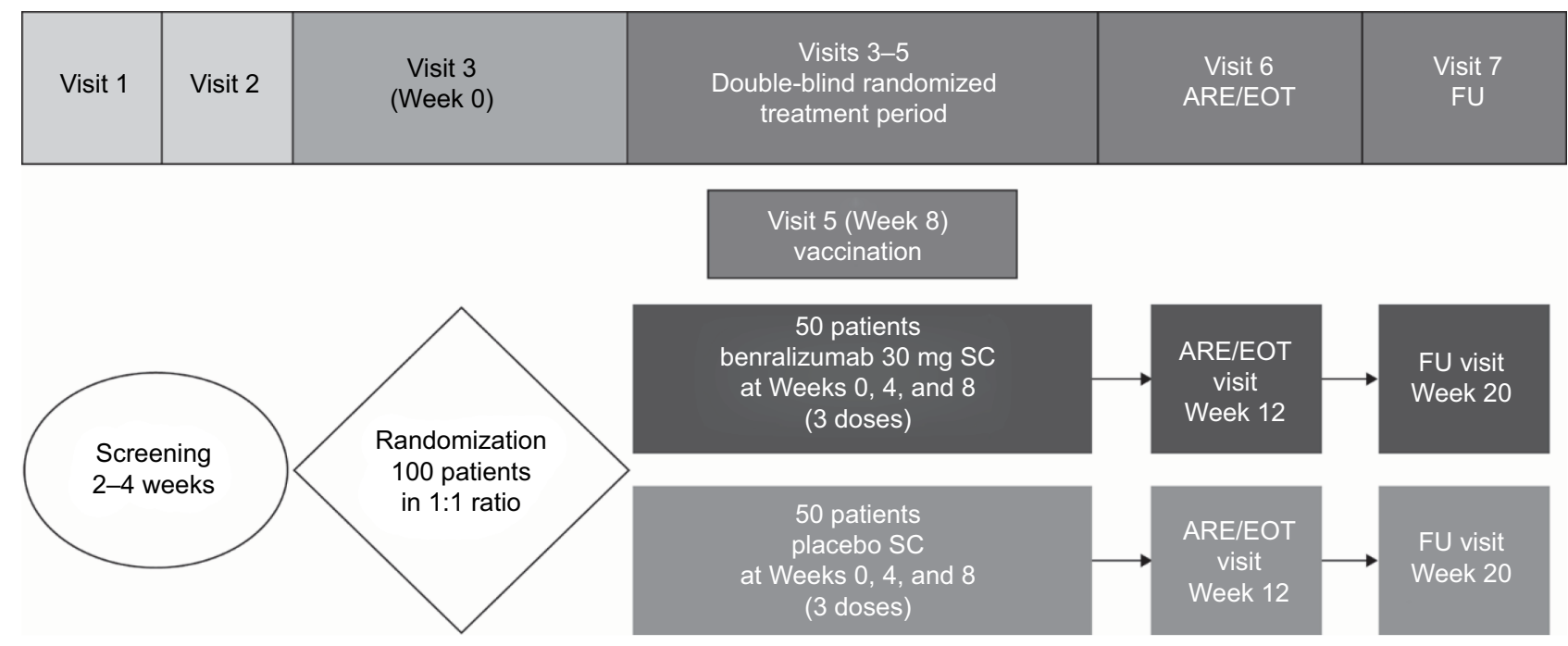

Figure I Study design.

Abbreviations: ARE, antibody response evaluation; EOT, end of treatment; FU, follow-up; SC, subcutaneous. 
virus vaccination, was assessed by post-dose, strain-specific hemagglutination inhibition (HAI) antibody geometric mean fold rises (GMFRs) from Week 8 to Week 12; post-dose, strain-specific HAI antibody geometric mean titers (GMTs) at Week 12; percentage of patients experiencing a post-dose, strain-specific HAI antibody response at Week 12 (defined as $\mathrm{a} \geq 4$-fold rise in HAI antibody titer from Week 8 to Week 12); and percentage of patients with a post-dose, strain-specific HAI antibody titer $\geq 40$ at Week 12 .

Secondary outcomes included percentage of patients achieving a post-dose, strain-specific HAI antibody titer $\geq 320$ at Week 12; post-dose, strain-specific microneutralization (MN) GMFRs from Week 8 to Week 12; post-dose, strainspecific MN antibody GMTs at Week 12; and percentage of patients experiencing a post-dose, strain-specific $\mathrm{MN}$ antibody response at Week 12 (defined as a $\geq 4$-fold rise in $\mathrm{MN}$ antibody titer from Week 8 to Week 12). Further secondary outcomes included ACQ-6 mean score change from baseline to Week 12, PK parameters, and safety (adverse events [AEs], serious adverse events [SAEs], and anti-drug antibody [ADAb] assessment).

\section{Analyses}

Strain-specific antibody responses were assessed by HAI and MN antibody assay. The HAI assay (Focus Diagnostics, Cypress, CA, USA) was designed to detect and measure HAI antibodies in test serum against the four influenza antigens in the FluLaval ${ }^{\circledR}$ Quadrivalent vaccine. This test utilizes the presence of HA, a protein that binds to sialic acid-containing receptors on the surface of red blood cells (RBCs), within the influenza virus. The presence of antibodies to antigenic determinants on the HA protein inhibits the binding between the viral HA protein and receptors on the RBC membrane, forming the basis of the assay. All procedures were performed according to the manufacturer's instructions. In brief, serum samples were treated with receptor-destroying enzyme overnight, diluted 1:10, and serial diluted 2-fold in triplicate from $1: 10$ to $1: 10,240$. An equal volume of standardized virus (4 HA unit $/ 25 \mu \mathrm{L}$ ) was added and neutralization occurred for 1 hour at $37^{\circ} \mathrm{C}$, followed by the addition of RBCs. Plates were tilted and hemagglutination pattern was observed. Antibody titer was determined as the reciprocal of the last dilution that fully inhibited hemagglutination compared with an RBC control.

The MN antibody assay was based on the inhibition of cytopathic effect formation in Madin-Darby canine kidney (MDCK) cells. In brief, serial diluted serum was pre-incubated (in duplicate from 1:10 to 1:5,120) with a standardized amount of virus (100 median tissue culture infective dose/50 $\mu \mathrm{L}$ ). MDCK cells were added and the plates incubated overnight (18-22 hours). The cells were fixed and the presence of virus was detected using an enzyme-linked immunosorbent assay with an antinucleoprotein antibody to influenza. The virus neutralizing antibody endpoint titer was determined using a formula that calculated the midpoint OD of uninfected cells and virus-infected (without neutralization) cells.

Benralizumab serum concentrations were analyzed by a central laboratory using a validated bioanalytical method. The PK assessment was primarily based on the observed steady-state serum trough (pre-dose) concentrations, $\mathrm{C}_{\text {trough }}$. A three-tiered testing plan, consisting of validated screening, confirmatory, and titer assays, was used to analyze serum samples for ADAb status. Empirical evaluation of potential impact of demographic covariates and $\mathrm{ADAb}$ on $\mathrm{C}_{\text {trough }}$ was conducted.

Data analyses were conducted using the $\mathrm{SAS}^{\circledR}$ System (SAS Institute Inc., Cary, NC, USA). The analysis of antibody endpoints (strain-specific GMFRs and GMTs) was summarized by descriptive statistics, geometric standard deviation (GSD), and geometric coefficient of variation (CV\%). For GMFR and GMT, the geometric least squares (LS) mean ratio between groups (placebo/benralizumab) along with the corresponding 90\% CI was calculated via an analysis of covariance (ANCOVA) model on log-transformed variables (antibody titer fold rise from Week 8 or antibody titer at Week 12). The linear model effects were treatment group as a fixed effect and age group (adolescents and young adults) as a fixed categorical covariate.

The model-based natural log estimates of the LS means for each treatment and the differences between the LS means (placebo-benralizumab) along with the corresponding $90 \%$ CI were exponentiated.

The percentage of patients who experienced a post-dose HAI or MN antibody response at Week 12 (defined as a $\geq 4$-fold rise in HAI or MN antibody titer from Week 8 to Week 12), post-dose HAI antibody titer $\geq 40$ at Week 12 , or post-dose HAI antibody titer $\geq 320$ at Week 12, and the corresponding 90\% Clopper-Pearson exact CIs were summarized by treatment for each outcome.

A post hoc sensitivity analysis was performed on the primary efficacy outcomes using the same statistical methods described previously, but excluding patients who received placebo who had a PK anomaly with serum benralizumab concentration $\geq 30 \mathrm{ng} / \mathrm{mL}$.

ACQ-6 score and change from baseline were summarized by visit and treatment group using descriptive statistics. 
Asthma control response status, as determined by the ACQ-6, was summarized at baseline and Week 12 by treatment group. Well controlled was defined as an end-of-treatment ACQ-6 score of $\leq 0.75$, partly controlled as $>0.75$ to $<1.5$, and not well controlled as $\geq 1.5$.

\section{Results}

\section{Patient population}

Between July 1, 2016, and January 24, 2017, 133 patients were screened. Of those, 103 were randomized at 30 study centers in the USA. Failed screening was the most common reason for not being randomized (18 patients); other reasons included being lost to follow-up (Figure 2). Of the randomized patients, $72(69.9 \%)$ were adolescents and 31 (30.1\%) were young adults (full analysis set: benralizumab, $\mathrm{n}=51$; placebo, $\mathrm{n}=52)$. Thirty-six $(70.6 \%)$ adolescent patients received benralizumab and $36(69.2 \%)$ received placebo. Of the young adults, $15(29.4 \%)$ and $16(30.8 \%)$ received benralizumab and placebo, respectively.

The vaccine immunogenicity analysis set included all randomized patients who received at least one dose of influenza vaccine plus one dose of benralizumab, had Week 8 and Week 12 data and no significant protocol deviations. The vaccine immunogenicity analysis set included $96.1 \%$ of randomized patients who completed the study (benralizumab, $n=50$; placebo, $n=49$ [Figure 2]). The safety analysis set comprised all patients who received at least one dose of study treatment (benralizumab, $\mathrm{n}=51$; placebo, $\mathrm{n}=52$ ). The $\mathrm{PK}$ analysis set comprised all patients who received benralizumab and for whom at least one quantifiable PK blood sample was obtained post-first dose (benralizumab, $\mathrm{n}=50$; placebo, $\mathrm{n}=51$ ).

Patient demographics and clinical characteristics were similar between treatment groups and representative of the intended target population (Table 1).

\section{Primary outcome}

Baseline HAI antibody GMTs at Week 8 were reasonably well-balanced across the two treatment groups, with greater titers for the A strains compared with the B strains. No consistent differences occurred in strain-specific HAI antibody responses at Week 12 for patients who received benralizumab vs placebo (Table 2). The GMFRs of HAI antibody titers

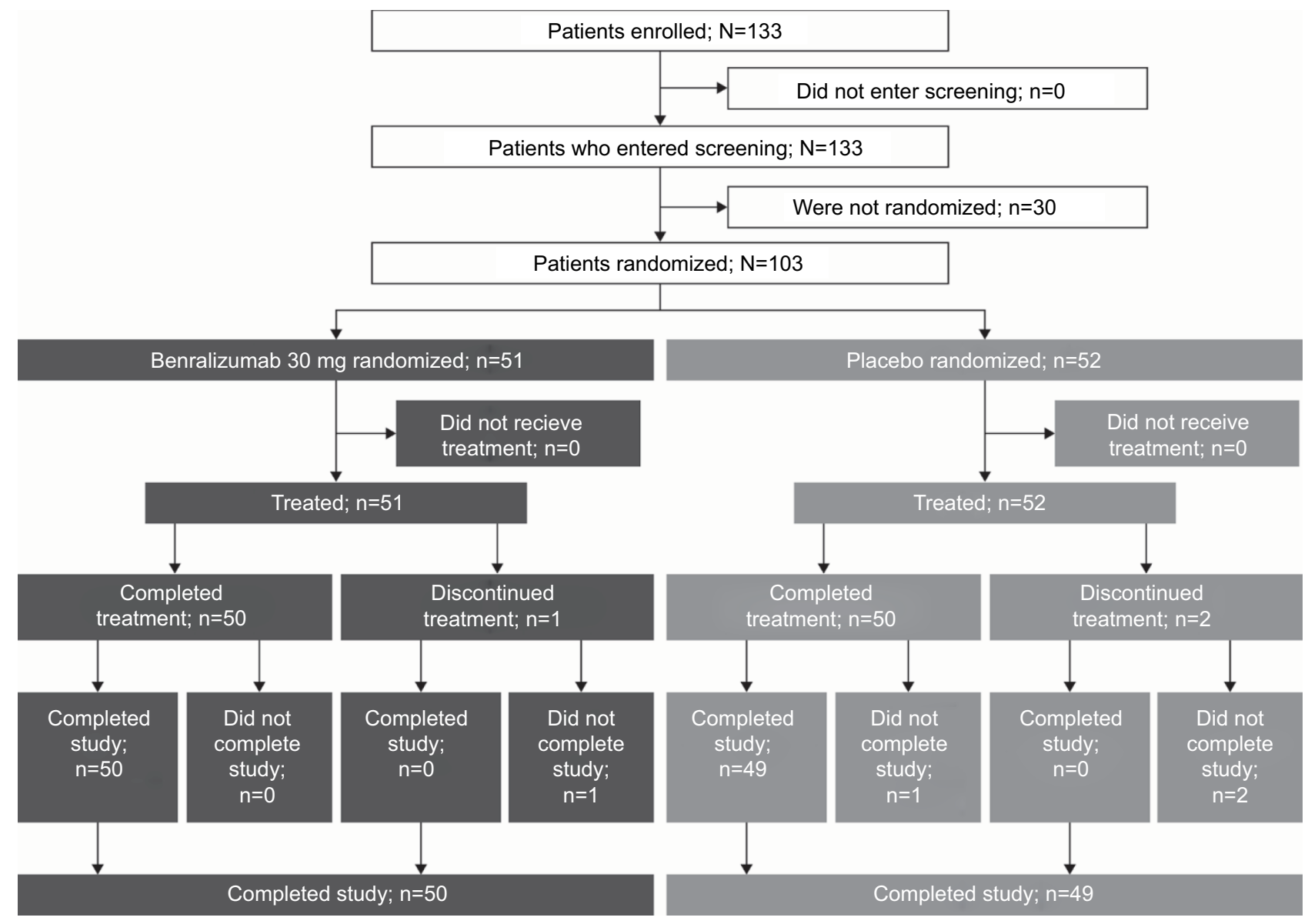

Figure 2 Consolidated standards of reporting (CONSORT) flow diagram. 
Table I Patient demographics and baseline clinical characteristics (full analysis set)

\begin{tabular}{|c|c|c|c|}
\hline & $\begin{array}{l}\text { Benralizumab } \\
30 \mathrm{mg} \text { Q4W } \\
(\mathrm{N}=5 \mathrm{I})\end{array}$ & $\begin{array}{l}\text { Placebo } \\
(N=52)\end{array}$ & $\begin{array}{l}\text { Total } \\
(N=103)\end{array}$ \\
\hline Age [years], median (range) & $16(|2-2|)$ & $16(|2-2|)$ & $16(|2-2|)$ \\
\hline Female, n (\%) & $2 I(4 \mid .2)$ & $21(40.4)$ & $42(40.8)$ \\
\hline \multicolumn{4}{|l|}{ Race, n (\%) } \\
\hline White & $38(74.5)$ & $36(69.2)$ & $74(7 \mathrm{I} .8)$ \\
\hline American Indian or Alaska Native & $0(0)$ & I (I.9) & I (I.0) \\
\hline Black or African-American & $13(25.5)$ & $13(25.0)$ & $26(25.2)$ \\
\hline Other & $0(0)$ & $2(3.8)$ & $2(1.9)$ \\
\hline BMI $\left[\mathrm{kg} / \mathrm{m}^{2}\right]$, mean $(\mathrm{SD})$ & $25.4(7.0)$ & $23.9(5.1)$ & $24.6(6.1)$ \\
\hline \multicolumn{4}{|l|}{ Asthma triggers, $\mathrm{n}(\%)$} \\
\hline Allergens & $42(82.4)$ & $44(84.6)$ & $86(83.5)$ \\
\hline Aspirin & $0(0)$ & $0(0)$ & $0(0)$ \\
\hline Exercise & $34(66.7)$ & $39(75.0)$ & $73(70.9)$ \\
\hline Other & $16(31.4)$ & $24(46.2)$ & $40(38.8)$ \\
\hline \multicolumn{4}{|l|}{ Exacerbations during the previous } \\
\hline \multicolumn{4}{|l|}{12 months, $\mathrm{n}(\%)$} \\
\hline 0 & $32(62.7)$ & $33(63.5)$ & $65(63.1)$ \\
\hline 1 & II (2I.6) & $12(23.1)$ & $23(22.3)$ \\
\hline$\geq 2$ & $8(15.7)$ & $7(13.4)$ & $15(14.6)$ \\
\hline 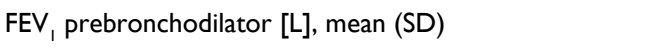 & $2.854(0.675)$ & $2.622(0.665)$ & $2.737(0.677)$ \\
\hline $\mathrm{FEV}$, prebronchodilator [\% predicted normal], mean (SD) & $79.91(14.15)$ & $76.79(14.45)$ & $78.34(14.32)$ \\
\hline $\mathrm{FEV}_{1} / \mathrm{FVC}$ prebronchodilator [\%], mean (SD) & $75.31(8.87)$ & $75.55(10.37)$ & $75.43(9.61)$ \\
\hline FEV, reversibility [\%], median (range) & $14.98(-9.25-44.00)$ & $18.00(-2.24-48.5 \mathrm{I})$ & $16.06(-9.25-48.51)$ \\
\hline
\end{tabular}

Abbreviations: BMI, body mass index; $\mathrm{FEV}_{1}$, forced expiratory volume in I second; FVC, forced vital capacity; $\mathrm{N}$, number of patients within each treatment group; $\mathrm{n}$, number of patients in specified subgroup; Q4W, every 4 weeks; SD, standard deviation.

from Weeks 8-12 for all influenza strains tested ranged from 3.3-4.2 for patients receiving benralizumab and 3.4-3.9 for those receiving placebo (Table 2, Figure 3 ). The estimated LS mean ratios for GMT and GMFR HAI antibody titers were similar between patients receiving benralizumab or placebo (3.3-4.1 and 3.1-3.9, respectively) (Table 2).

$\mathrm{A} \geq 4$-fold rise in strain-specific HAI antibodies from Week 8 to Week 12 occurred in $44.0 \%-56.0 \%$ and $30.6 \%-49.0 \%$ of patients receiving benralizumab and placebo, respectively, (Table 2). At Week 12, 78.0\%-100.0\% vs 79.6\%-100.0\% of patients receiving benralizumab and placebo, respectively, achieved an HAI antibody titer $\geq 40$ (Table 2).

Comparable results were seen in the sensitivity analyses for the primary endpoints that excluded the patient with PK anomalies.

\section{Antibody response secondary outcomes}

At Week 12, the percentage of patients who experienced HAI antibody responses $\geq 320$ was greater for influenza A strains than influenza B strains (Table 2). Between $50.0 \%-84.0 \%$ of patients receiving benralizumab, and $61.2 \%-85.7 \%$ of patients receiving placebo, achieved $a \geq 320$ HAI antibody titer for influenza A strains. For influenza B strains, $2.0 \%-8.0 \%$ and $2.0 \%-4.1 \%$ of patients receiving benralizumab and placebo, respectively, achieved a $\geq 320$ HAI antibody titer.

Baseline MN antibody GMTs at Week 8 were also comparatively well-balanced across the two treatment groups, with greater titers for the A strains compared with the B strains. There were no consistent differences in MN strain-specific antibody responses at Week 12 between patients receiving benralizumab or placebo (Table 3 ). The MN antibody GMFRs from Week 8 to Week 12 for all influenza strains tested ranged from 2.8-5.1 for patients receiving benralizumab and 3.2-4.4 for those receiving placebo (Table 3 , Figure 3 ). A $\geq 4$-fold rise in $\mathrm{MN}$ antibodies from Week 8 to Week 12 occurred in $28.0 \%-44.0 \%$ and $38.8 \%-42.9 \%$ of patients receiving benralizumab and placebo, respectively (Table 3 ).

\section{Patient-reported secondary outcomes}

Improvement in ACQ-6 scores was noted at Week 4 and maintained through Week 12 for patients receiving benralizumab or placebo. Mean score changes were -0.50 and -0.42 for patients receiving benralizumab and placebo, respectively (Table 4). There was no clear difference between benralizumab and placebo groups in the percentage 
Table 2 Influenza strain antibody response for hemagglutination inhibition (vaccine immunogenicity analysis set)

\begin{tabular}{|c|c|c|}
\hline Antibody response measure & $\begin{array}{l}\text { Benralizumab } 30 \mathrm{mg} \text { Q4W } \\
(\mathrm{N}=50)\end{array}$ & $\begin{array}{l}\text { Placebo } \\
(\mathrm{N}=49)\end{array}$ \\
\hline \multicolumn{3}{|l|}{ GMT Week 8, GM (GSD; CV\%) } \\
\hline Influenza A, HINI & $145.9(3.6 ; 205.1)$ & $166.9(2.8 ; 136.6)$ \\
\hline Influenza A, H3N2 & $62.3(3.7 ; 209.6)$ & $67.2(3.4 ; 185.3)$ \\
\hline Influenza B, Yamagata lineage & $16.6(2.6 ; 122.6)$ & $18.5(2.6 ; 123.5)$ \\
\hline Influenza B, Victoria lineage & $13.6(2.5 ; 1 \mid 1.2)$ & $21.4(3.2 ; 167.1)$ \\
\hline \multicolumn{3}{|c|}{ GMT Week I2, GM (GSD; CV\%) } \\
\hline Influenza A, HINI & $570.2(2.4 ; 107.9)$ & $566.1(2.2 ; 93.2)$ \\
\hline Influenza A, H3N2 & $206.3(2.9 ; 146.6)$ & $263.7(2.7 ; 126.7)$ \\
\hline Influenza B, Yamagata lineage & $60.3(2.2 ; 93.1)$ & $62.0(2.2 ; 93.2)$ \\
\hline Influenza B, Victoria lineage & $57.9(2.7 ; 126.2)$ & $72.8(2.3 ; 99.4)$ \\
\hline \multicolumn{3}{|c|}{ GMFR Week 8 to Week I2, GM (GSD; CV\%) } \\
\hline Influenza A, HINI & $3.9(4.1 ; 251.9)$ & $3.4(3.5 ; 191.3)$ \\
\hline Influenza A, H3N2 & $3.3(2.8 ; 136.2)$ & $3.9(3.2 ; 168.6)$ \\
\hline Influenza B, Yamagata lineage & $3.6(2.9 ; 144.9)$ & $3.4(2.5 ; 115.0)$ \\
\hline Influenza B, Victoria lineage & $4.2(3.3 ; 178.7)$ & $3.4(3.1 ; 162.2)$ \\
\hline \multicolumn{3}{|c|}{ GMT Week 12 geometric LS mean estimate (LS mean ratio; $90 \% \mathrm{Cl}^{\mathrm{a}}$ ) } \\
\hline Influenza A, HINI & $521.1(1.00 ; 0.76-1.31)$ & 518.6 \\
\hline Influenza A, H3N2 & I70.7 (I.28; 0.93-I.77) & 219.4 \\
\hline Influenza B, Yamagata lineage & $61.5(1.03 ; 0.79-1.34)$ & 63.2 \\
\hline Influenza B, Victoria lineage & $53.1(1.26 ; 0.93-1.70)$ & 66.8 \\
\hline \multicolumn{3}{|c|}{ GMFR Week I 2 geometric LS mean estimate (LS mean ratio; $90 \% \mathrm{Cl}^{2}$ ) } \\
\hline Influenza A, HINI & $3.6(0.87 ; 0.56-1.35)$ & 3.1 \\
\hline Influenza A, H3N2 & $3.3(1.19 ; 0.82-1.71)$ & 3.9 \\
\hline Influenza B, Yamagata lineage & $3.4(0.93 ; 0.67-1.29)$ & 3.2 \\
\hline Influenza B, Victoria lineage & $4.1(0.8 ; 0.54-1.19)$ & 3.3 \\
\hline \multicolumn{3}{|c|}{ Antibody titer $\geq 4$-fold rise from Week 8 to Week I2, n (90\% Cl'b $[\%]$} \\
\hline Influenza A, HINI & $22(0.32-0.57)[44.0]$ & $15(0.20-0.43)[30.6]$ \\
\hline Influenza A, H3N2 & $25(0.38-0.62)[50.0]$ & $24(0.37-0.62)[49.0]$ \\
\hline Influenza B, Yamagata lineage & $24(0.36-0.60)[48.0]$ & $24(0.37-0.62)[49.0]$ \\
\hline Influenza B, Victoria lineage & $28(0.43-0.68)[56.0]$ & $20(0.29-0.54)[40.8]$ \\
\hline \multicolumn{3}{|c|}{ Antibody titer $\geq 40$ at Week I $2, \mathrm{n}\left(90 \% \mathrm{Cl}^{\mathrm{b}}\right)$ [\%] } \\
\hline Influenza A, HINI & $50(0.94-1.00)[100.0]$ & $49(0.94-1.00)[100.0]$ \\
\hline Influenza A, H3N2 & $49(0.91-1.00)[98.0]$ & $48(0.91-1.00)[98.0]$ \\
\hline Influenza B, Yamagata lineage & $43(0.75-0.93)[86.0]$ & $39(0.68-0.88)[79.6]$ \\
\hline Influenza B, Victoria lineage & $39(0.66-0.87)[78.0]$ & $43(0.77-0.95)[87.8]$ \\
\hline \multicolumn{3}{|c|}{ Antibody titer $\geq 320$ at Week I $2, \mathrm{n}\left(90 \% \mathrm{Cl}^{\mathrm{b}}\right)$ [\%] } \\
\hline Influenza A, HINI & $42(0.73-0.92)[84.0]$ & $42(0.75-0.93)[85.7]$ \\
\hline Influenza A, H3N2 & $25(0.38-0.62)[50.0]$ & $30(0.48-0.73)[61.2]$ \\
\hline Influenza B, Yamagata lineage & I $(0.00-0.09)[2.0]$ & I $(0.00-0.09)[2.0]$ \\
\hline Influenza B, Victoria lineage & $4(0.03-0.17)[8.0]$ & $2(0.01-0.12)[4.1]$ \\
\hline
\end{tabular}

Notes: ${ }^{9} 90 \%$ Cls are for treatment comparison between benralizumab and placebo. ${ }^{\circ}$ Results using the Clopper-Pearson method.

Abbreviations: CV\%, geometric coefficient of variation; GM, geometric mean; GMFR, geometric mean fold rise; GMT, geometric mean titer; GSD, geometric standard deviation; LS, least squares; N, number of patients within each treatment group; n, number of patients in analysis; Q4W, every 4 weeks.

of patients who were not well controlled (ACQ-6 $\geq 1.5$ ) at Week 12 (benralizumab, $\mathrm{n}=18$ [35.3\%]; placebo, $\mathrm{n}=19$ $[36.5 \%])$.

\section{Pharmacokinetics}

Serum concentrations were quantifiable in all benralizumabtreated patients at Week 12, with a geometric mean value of $1,323.6 \mathrm{ng} / \mathrm{mL}$ (129.99 CV\%). Based on the geometric means, PK steady state for the benralizumab group was reached by Week 8; the lower limit of quantification was $3.86 \mathrm{ng} / \mathrm{mL}$ (Figure 4).

\section{Eosinophil depletion}

Mean eosinophil counts at baseline were similar for patients in the benralizumab and placebo groups $\left(0.254 \times 10^{9}\right.$ cells $/ \mathrm{L}$ and $0.221 \times 10^{9}$ cells $/ \mathrm{L}$, respectively). By Week 12 , mean eosinophil count had decreased to $0.028 \times 10^{9}$ cells $/ \mathrm{L}$ in the benralizumab group, compared with a mean of $0.288 \times 10^{9}$ cells $/ \mathrm{L}$ 

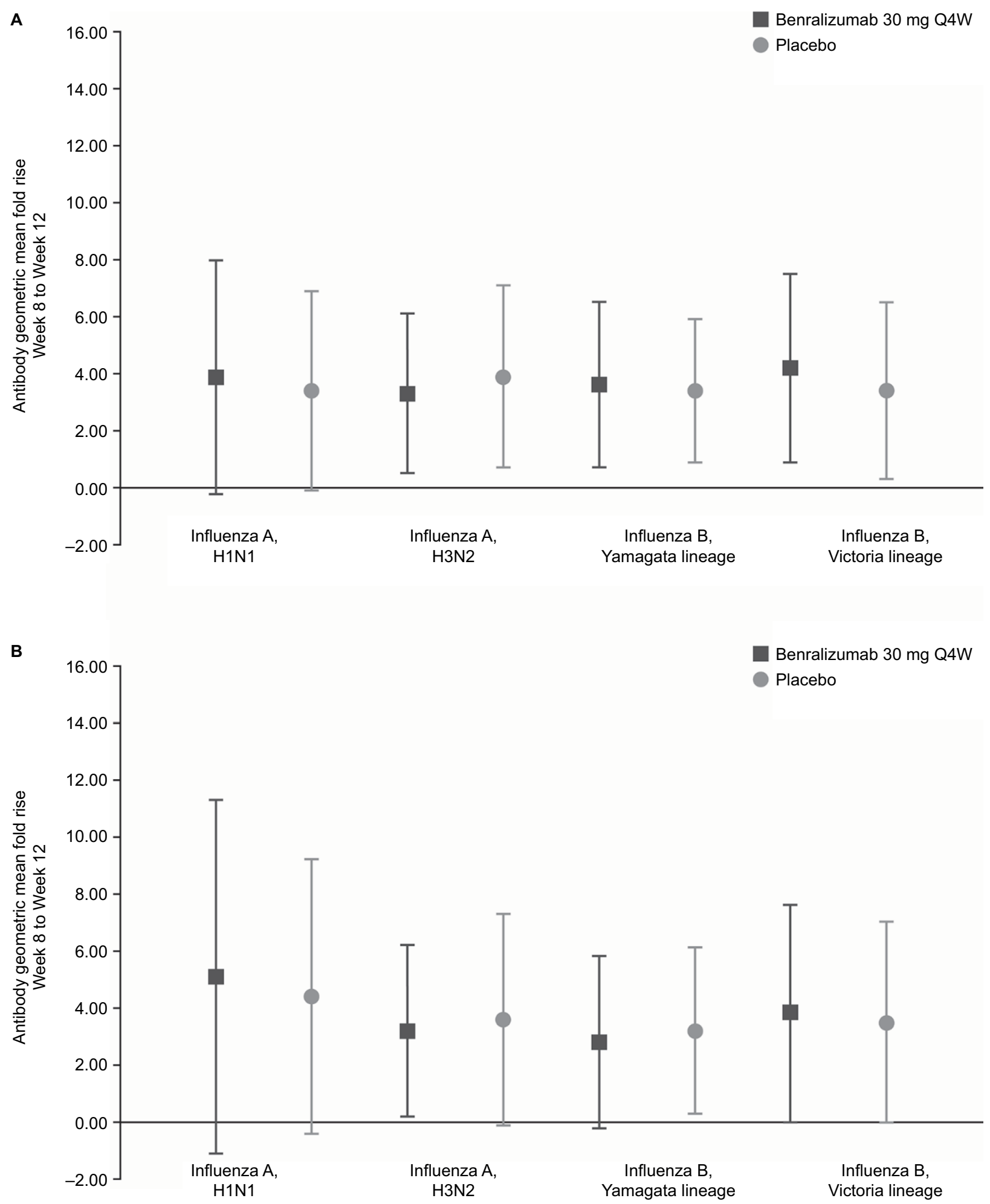

Figure 3 Influenza strain antibody geometric mean fold rise from Week 8 to Week 12 for (A) hemagglutination inhibition and (B) microneutralization (vaccine immunogenicity analysis set).

Note: Error bars represent geometric SD.

Abbreviation: Q4W, every 4 weeks.

in the placebo group. At Week 20, mean eosinophil count remained low in the benralizumab group $\left(0.055 \times 10^{9}\right.$ cells $\left./ \mathrm{L}\right)$ compared with the placebo group $\left(0.324 \times 10^{9}\right.$ cells/L).

\section{Anti-drug antibody analysis}

Overall, eight patients (15.7\%) were found to be positive for ADAbs to benralizumab at any visit (Week 0, 8, 12, 
Table 3 Influenza strain antibody response for microneutralization (vaccine immunogenicity analysis set)

\begin{tabular}{|c|c|c|}
\hline Antibody response measure & $\begin{array}{l}\text { Benralizumab } 30 \mathrm{mg} \text { Q4W } \\
(\mathrm{N}=50)\end{array}$ & $\begin{array}{l}\text { Placebo } \\
(N=49)\end{array}$ \\
\hline \multicolumn{3}{|l|}{ GMT Week 8, GM (GSD; CV\%) } \\
\hline Influenza A, HINI & $735.2(4.2 ; 263.0)$ & $898.7(3.4 ; 188.9)$ \\
\hline Influenza A, H3N2 & $\mathrm{I}, 353.0(2.5 ; \mathrm{I} \mid 4.7)$ & $\mathrm{I}, 2 \mathrm{I} 8.2(2.7 ; \mathrm{I} 32.2)$ \\
\hline Influenza B, Yamagata lineage & $123.0(2.7 ; 129.8)$ & $106.2(2.7 ; 129.4)$ \\
\hline Influenza B, Victoria lineage & $43.7(2.8 ; 138.4)$ & $67.0(3.5 ; 196.8)$ \\
\hline \multicolumn{3}{|c|}{ GMT Week I 2, GM (GSD; CV\%) } \\
\hline Influenza A, HINI & $3,774.1(3.4 ;|8| .7)$ & $3,969.1(3.0 ; 154.0)$ \\
\hline Influenza A, H3N2 & $4,307.5(3.2 ; 169.0)$ & $4,351.3(3.2 ;|7| .0)$ \\
\hline Influenza B, Yamagata lineage & $350.2(2.4 ; 103.6)$ & $336.2(2.5 ; 114.3)$ \\
\hline Influenza B, Victoria lineage & $164.5(3.3 ; 178.5)$ & $234.4(2.8 ; 135.0)$ \\
\hline \multicolumn{3}{|c|}{ GMFR Week 8 to Week I2, GM (GSD; CV\%) } \\
\hline Influenza A, HINI & $5.1(6.2 ; 521.4)$ & $4.4(4.8 ; 329.4)$ \\
\hline Influenza A, H3N2 & $3.2(3.0 ; 149.8)$ & $3.6(3.7 ; 210.0)$ \\
\hline Influenza B, Yamagata lineage & $2.8(3.0 ; 148.9)$ & $3.2(2.9 ; 141.3)$ \\
\hline Influenza B, Victoria lineage & $3.8(3.8 ; 224.1)$ & $3.5(3.5 ; 195.3)$ \\
\hline \multicolumn{3}{|c|}{ Antibody titer $\geq 4$-fold rise from Week 8 to Week I2, n (90\% Cla) [\%] } \\
\hline Influenza A, HINI & $21(0.30-0.55)[42.0]$ & $20(0.29-0.54)[40.8]$ \\
\hline Influenza $\mathrm{A}, \mathrm{H} 3 \mathrm{~N} 2$ & $22(0.32-0.57)[44.0]$ & $21(0.31-0.56)[42.9]$ \\
\hline Influenza B, Yamagata lineage & $14(0.18-0.40)[28.0]$ & $19(0.27-0.52)[38.8]$ \\
\hline Influenza B, Victoria lineage & $20(0.28-0.53)[40.0]$ & $19(0.27-0.52)[38.8]$ \\
\hline
\end{tabular}

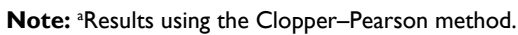

Abbreviations: CV\%, geometric coefficient of variation; GM, geometric mean; GMFR, geometric mean fold rise; GMT, geometric mean titer; GSD, geometric standard deviation; $\mathrm{N}$, number of patients within each treatment group; $n$, number of patients in analysis; Q4W, every 4 weeks.

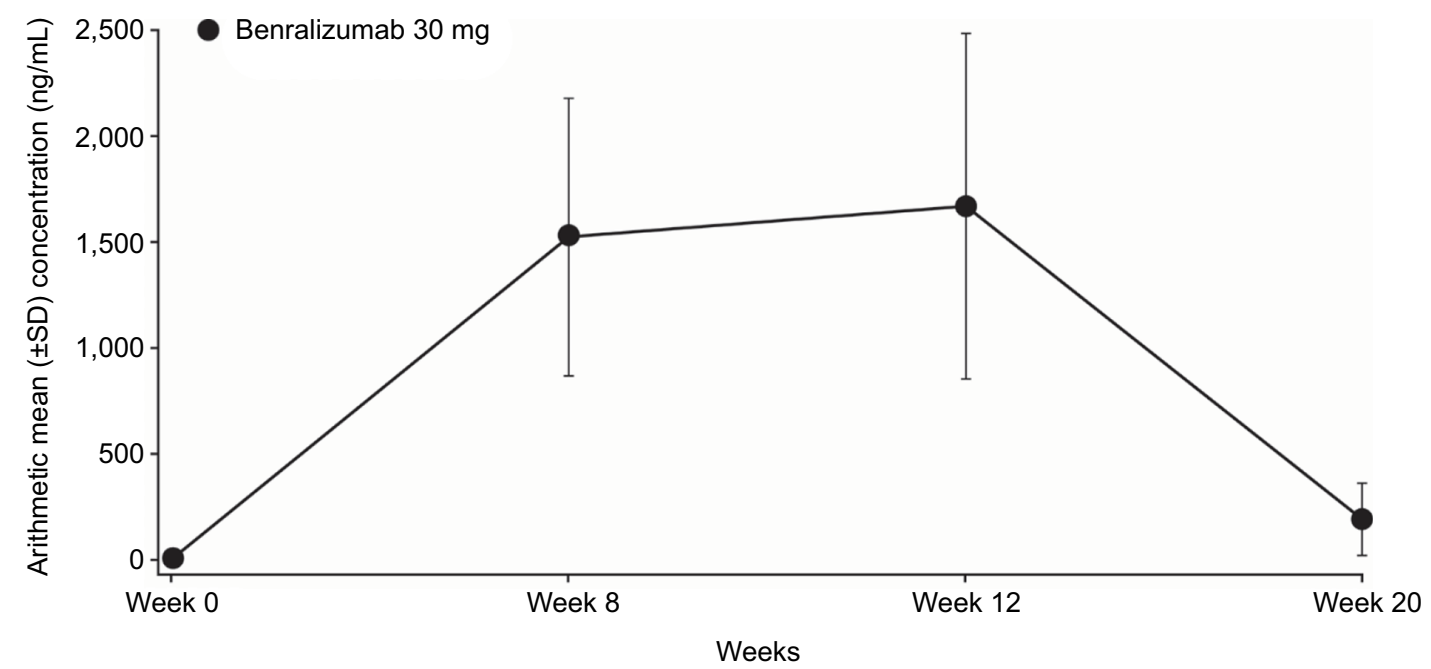

Figure 4 Arithmetic mean $( \pm S D)$ serum trough concentrations of benralizumab (PK analysis set).

Abbreviations: PK, pharmacokinetics; SD, standard deviation.

or 20). Two patients were ADAb positive at baseline, four patients became ADAb positive by Week 12 , and two patients became $\mathrm{ADAb}$ positive by Week 20 . In all eight patients, blood eosinophil counts were depleted at vaccine assessment (Week 12), indicating that sufficient benralizumab was present to maintain its pharmacodynamic effect.

\section{Safety}

The number of patients with AEs was similar in the benralizumab and placebo groups (Table 5). SAEs were infrequent, and most AEs reported were mild or moderate. No deaths were reported. Incidence of the most common AEs (those occurring at a frequency of $\geq 3 \%$ in any group) was similar between treatment groups and included asthma, headache, nasopharyngitis, oropharyngeal pain, viral gastroenteritis, and upper respiratory tract infection (Table 5).

\section{Discussion}

Immunomodulators may impact vaccine immune response; therefore, it is important to determine whether treatment 
Table 4 ACQ-6 scores by time point (full analysis set)

\begin{tabular}{|c|c|c|c|c|c|c|}
\hline \multirow[t]{2}{*}{ Time point } & \multicolumn{3}{|c|}{$\begin{array}{l}\text { Benralizumab } 30 \mathrm{mg} \text { Q4W } \\
(\mathrm{N}=5 \mathrm{I})\end{array}$} & \multicolumn{3}{|c|}{$\begin{array}{l}\text { Placebo } \\
(\mathrm{N}=52)\end{array}$} \\
\hline & $\mathbf{n}$ & Score & Change from baseline & $\mathbf{n}$ & Score & Change from baseline \\
\hline Baseline, mean (SD) & 51 & $1.95(0.88)$ & & 52 & $\mathrm{I} .76(0.95)$ & \\
\hline Week 4, mean (SD) & 51 & $1.47(0.98)$ & $-0.48(0.98)$ & 50 & $\mathrm{I} .44(0.88)$ & $-0.30(0.74)$ \\
\hline Week 8, mean (SD) & 50 & $1.47(0.94)$ & $-0.47(0.89)$ & 50 & I.34 (0.78) & $-0.39(0.87)$ \\
\hline Week I2, mean (SD) & 50 & $1.44(1.06)$ & $-0.50(\mathrm{I} .1 \mathrm{I})$ & 49 & $1.29(0.83)$ & $-0.42(0.93)$ \\
\hline
\end{tabular}

Abbreviations: ACQ-6, Asthma Control Questionnaire 6; N, number of patients within each treatment group; n, number of patients in analysis; Q4W, every 4 weeks; SD, standard deviation.

Table 5 Adverse events (safety analysis set)

\begin{tabular}{lll}
\hline AE category, $\mathbf{n}(\%)$ & $\begin{array}{l}\text { Benralizumab } \\
\mathbf{3 0} \mathbf{~ m g ~ Q 4 W ~} \\
\mathbf{( N = 5 1 )}\end{array}$ & $\begin{array}{l}\text { Placebo } \\
\mathbf{( N = 5 2 )}\end{array}$ \\
\hline Any AE & $22(43.1)$ & $23(44.2)$ \\
Any SAE (including events with outcome & $0(0)$ & $2(3.8)$ \\
of death) & & \\
Any AE of maximum intensity & $10(19.6)$ & $12(23.1)$ \\
$\quad$ Mild & $9(17.6)$ & $10(19.2)$ \\
$\quad$ Moderate & $3(5.9)$ & $1(1.9)$ \\
$\quad$ Severe & $1(2.0)$ & $2(3.8)$ \\
Any causally related AE & $3(5.9)$ & $4(7.7)$ \\
Asthma & $2(3.9)$ & $4(7.7)$ \\
Headache & $2(3.9)$ & $4(7.7)$ \\
Nasopharyngitis & $3(5.9)$ & $2(3.8)$ \\
Oropharyngeal pain & $3(5.9)$ & $\mathrm{I}(1.9)$ \\
Viral gastroenteritis & $3(5.9)$ & $\mathrm{I}(1.9)$ \\
Upper respiratory tract infection &
\end{tabular}

Notes: Patients with several events in the same category are counted only once in that category. Patients with events in more than one category are counted once in each of those categories.

Abbreviations: $A E$, adverse event; $N$, number of patients within each treatment group; n, number of patients in subgroup; Q4W, every 4 weeks; SAE, serious AE.

with the eosinophil-depleting drug benralizumab influences immune system functioning following vaccination. ${ }^{14}$ Vaccines remained immunogenic for patients receiving immunomodulators in studies mostly of patients with rheumatic diseases, but the response may be attenuated with certain drugs. ${ }^{14}$ An in vivo study in mice reported that treatment with anti-IL-5 antibodies does not impede the immune response to vaccination. ${ }^{15}$ It is, however, important to confirm that treatment with the anti-IL-5R $\alpha$ antibody, benralizumab, does not negatively impact patients' immune responses.

Overall, our results demonstrate no consistent effects of treatment with benralizumab compared with placebo on the humoral antibody responses induced by seasonal influenza virus vaccination in adolescent and young adult patients with moderate to severe asthma. There were no consistent differences in the effects on the influenza strain-specific antibody responses for $\mathrm{HAI}$ or $\mathrm{MN}$ antibodies at Week 12 for patients in the benralizumab vs placebo groups, and the percentage of patients who experienced influenza strain-specific antibody responses with a $\geq 4$-fold rise from Week 8 to Week 12 for HAI and MN was similar across strains and between treatment groups. We selected this definition of adequate response based on the FluLaval ${ }^{\circledR}$ Quadrivalent prescribing information that defines seroconversion as a 4-fold increase in postvaccination antibody titer from pre-vaccination titer $\geq 1: 10$, or an increase in titer from $<1: 10$ to $\geq 1: 40 .{ }^{16}$ Although specific concentrations of HAI post-vaccination antibody titers within activated influenza virus vaccines have not been associated with protection from influenza, antibody titers have been used as a measure of vaccine activity. In some human challenge studies, antibody titers of $\geq 1: 40$ have been associated with protection from influenza in up to $50 \%$ of patients. ${ }^{17,18}$

Influenza vaccination is recommended for patients with asthma in many countries, because influenza infection is potentially associated with risk of asthma exacerbations. ${ }^{10}$ Children with asthma are more likely than healthy control children to have influenza-related outpatient physician visits or hospitalizations. ${ }^{10}$ However, data are limited on the clinical or serological effect of vaccination for patients with asthma in general and the effect of asthma treatments on patients' immune response to vaccination. ${ }^{19}$ Our results provide supportive evidence that treatment with the eosinophil-depleting biologic benralizumab does not impact the humoral immune response to vaccination for adolescent and young adult patients with moderate to severe eosinophilic asthma. This finding is consistent with the limited data available. Studies have reported that systemic corticosteroids did not impact immune response to influenza vaccination in children. ${ }^{10}$ In addition, treatment with biologics, high-dosage ICS, or systemic steroids did not significantly impact the immune response to pneumococcal polysaccharide vaccination for adult patients with asthma. ${ }^{19}$ However, there is some evidence that high-dosage ICS can reduce the immune response to vaccination against influenza $B{ }^{10}$ 
Secondary endpoints in the ALIZE trial included the impact of benralizumab on health-related quality of life, PK parameters, and safety of benralizumab in the adolescent and young adult population. In these patients, benralizumab treatment demonstrated a trend toward improved asthma control compared with placebo, as measured by the ACQ-6. However, improvement in ACQ-6 scores was noted at Week 4 and maintained through Week 12 for patients receiving benralizumab or placebo. This finding is consistent with results reported for the SIROCCO and CALIMA trials, in which adolescent patients receiving benralizumab or placebo showed improvements from baseline in all efficacy measures. However, efficacy was not confirmed in the adolescent population for the benralizumab treatment groups relative to placebo. ${ }^{5,6}$ This result may potentially be attributed to the small sample size and low exacerbation rate for adolescent patients receiving placebo who were enrolled in these trials.

PK analysis in this study confirmed that serum concentrations were quantifiable in all benralizumab-treated patients at Week 12, with a geometric mean value of $1,323.6 \mathrm{ng} / \mathrm{mL}$. Based on the geometric mean, PK steady state for the benralizumab group was reached at Week 8 . Further safety analyses found that the presence of ADAbs to benralizumab did not impact eosinophil counts at the time of the vaccine antibody response assessment. In addition, benralizumab was well-tolerated by patients in this study and no unexpected safety findings occurred, consistent with previous reports on benralizumab treatment for patients with severe asthma. ${ }^{5,6}$

\section{Conclusion}

In conclusion, benralizumab did not impair antibody response to seasonal virus vaccination in adolescents and young adult patients with moderate to severe asthma.

\section{Data availability}

Results of the ALIZE trial became available on August 24, 2017.

\section{Acknowledgments}

The authors would like to thank Joe Neubauer (AstraZeneca, Gaithersburg, MD, USA) and Michalina Marszewska (AstraZeneca, Warsaw, Poland) for their clinical operations leadership in this study. Editorial support was provided by Debra Scates, PhD, of JK Associates, Inc., and
Michael A. Nissen, ELS, of AstraZeneca and funded by AstraZeneca. The ALIZE trial was funded by AstraZeneca.

\section{Disclosures}

Pamela L Zeitlin has received consultation fees from AstraZeneca, Proteostasis Therapeutics Inc., and Shin Nippon Biomedical Laboratories and speaker fees from Clinical Insights Ltd. Raburn M Mallory is an employee of MedImmune LLC. Vivian H Shih, Richard F Olsson, and Mitchell Goldman are employees of AstraZeneca. The other authors report no conflicts of interest in this work.

\section{References}

1. Kolbeck R, Kozhich A, Koike M, et al. MEDI-563, a humanized anti-IL-5 receptor alpha $\mathrm{mAb}$ with enhanced antibody-dependent cell-mediated cytotoxicity function. JAllergy Clin Immunol. 2010;125(6):1344-1353.

2. Lötvall J, Pullerits T. Treating asthma with anti-IgE or anti-IL5. Curr Pharm Des. 1999;5(10):757-770.

3. Laviolette M, Gossage DL, Gauvreau G, et al. Effects of benralizumab on airway eosinophils in asthmatic patients with sputum eosinophilia. J Allergy Clin Immunol. 2013;132(5):1086-1096.

4. Busse WW, Katial R, Gossage D, et al. Safety profile, pharmacokinetics, and biologic activity of MEDI-563, an anti-IL-5 receptor alpha antibody, in a phase I study of subjects with mild asthma. J Allergy Clin Immunol. 2010;125(6):1237-1244.

5. Bleecker ER, FitzGerald JM, Chanez P, et al. Efficacy and safety of benralizumab for patients with severe asthma uncontrolled with highdosage inhaled corticosteroids and long-acting $\beta_{2}$-agonists (SIROCCO): a randomised, multicentre, placebo-controlled phase 3 trial. Lancet. 2016;388(10056):2115-2127.

6. FitzGerald JM, Bleecker ER, Nair P, et al. Benralizumab, an antiinterleukin-5 receptor $\alpha$ monoclonal antibody, as add-on treatment for patients with severe, uncontrolled, eosinophilic asthma (CALIMA): a randomised, double-blind, placebo-controlled phase 3 trial. Lancet. 2016;388(10056):2128-2141.

7. FASENRA ${ }^{\mathrm{TM}}$ (benralizumab) injection, for subcutaneous use [prescribing information]. Wilmington, DE: AstraZeneca Pharmaceuticals LP; 2017. Available from: https://www.accessdata.fda.gov/drugsatfda_docs/ label/2017/761070s000lbl.pdf. Accessed July 11, 2018.

8. FASENRA ${ }^{\mathrm{TM}}$ (benralizumab) injection, for subcutaneous use [summary of product characteristics]. Wilmington, DE: AstraZeneca Pharmaceuticals LP; 2018. Available from: http://ec.europa.eu/health/documents/ community-register/2018/20180108139598/anx_139598_en.pdf. Accessed July 11, 2018.

9. AstraZeneca. Fasenra receives approval in Japan [press release]. AstraZeneca; 2018. Available from: https://www.astrazeneca.com/media-centre/press-releases/2018/fasenra-recieves-approval-in-japan-19012018. html. Accessed July 11, 2018.

10. Pesek R, Lockey R. Vaccination of adults with asthma and COPD. Allergy. 2011;66(1):25-31.

11. Vasileiou E, Sheikh A, Butler C, et al. Effectiveness of influenza vaccines in asthma: a systematic review and meta-analysis. Clin Infect Dis. 2017;65(8):1388-1395.

12. Esnault S, Kelly EA. Essential mechanisms of differential activation of eosinophils by IL-3 compared to GM-CSF and IL-5. Crit Rev Immunol. 2016;36(5):429-444.

13. Jacobsen EA, Helmers RA, Lee JJ, Lee NA. The expanding role(s) of eosinophils in health and disease. Blood. 2012;120(19):3882-3890.

14. McMahan ZH, Bingham CO, 3rd. Effects of biological and non-biological immunomodulatory therapies on the immunogenicity of vaccines in patients with rheumatic diseases. Arthritis Res Ther. 2014;16(6):506. 
15. Sher A, Coffman RL, Hieny S, Cheever AW. Ablation of eosinophil and IgE responses with anti-IL-5 or anti-IL-4 antibodies fails to affect immunity against Schistosoma mansoni in the mouse. J Immunol. 1990;145(11):3911-3916.

16. FLULAVAL QUADRIVALENT (influenza vaccination) suspension for intramuscular injection [highlights of prescribing information]. Research Triangle Park, NC: GlaxoSmithKline; 2013. Available from: https://www.fda.gov/downloads/BiologicsBloodVaccines/Vaccines/ ApprovedProducts/UCM404086.pdf. Accessed July 11, 2018.
17. Hannoun C, Megas F, Piercy J. Immunogenicity and protective efficacy of influenza vaccination. Virus Res. 2004;103(1-2):133-138.

18. Hobson D, Curry RL, Beare AS, Ward-Gardner A. The role of serum haemagglutination-inhibiting antibody in protection against challenge infection with influenza A2 and B viruses. J Hyg. 1972;70(4):767-777.

19. Laratta CR, Williams K, Vethanayagam D, Ulanova M, Vliagoftis H. A case series evaluating the serological response of adult asthma patients to the 23-valent pneumococcal polysaccharide vaccine. Allergy Asthma Clin Immunol. 2017;13:27.

\section{Publish your work in this journal}

The Journal of Asthma and Allergy is an international, peer-reviewed open access journal publishing original research, reports, editorials and commentaries on the following topics: Asthma; Pulmonary physiology; Asthma related clinical health; Clinical immunology and the immunological basis of disease; Pharmacological interventions and new therapies. This journal is included in PubMed. The manuscript management system is completely online and includes a very quick and fair peer-review system, which is all easy to use. Visit http://www. dovepress.com/testimonials.php to read real quotes from published authors. 